\title{
PRODUTIVIDADE TOTAL DE FATORES E EFICIÊNCIA NO SETOR DE LAVOURAS DA AGRICULTURA BRASILEIRA ${ }^{1}$
}

José R. Vicente ${ }^{2}$

Resumo: Este estudo mensurou os níveis de produtividade total de fatores e de eficiências técnica, alocativa e econômica na produção agrícola do Brasil, setor de lavouras, em 2006. Um modelo de fronteira não-paramétrica (DEA) sob retornos constantes à escala foi utilizado. Os resultados sugeriram, em média, que o setor sofreu de ineficiência técnica moderada e de ineficiência alocativa forte. Se a eficiência técnica plena fosse atingida, a produção do setor de lavouras aumentaria 5\% sobre a obtida em 2006. Foi constatada sobreutilização de trabalho. A agricultura do estado de São Paulo era a única operando com eficiência econômica plena. Os dados indicaram um processo de convergência nos índices de produtividade e de eficiência em 2006, na comparação com 1995.

Palavras-chave: oferta agropecuária, produtividade total de fatores, eficiência econômica, eficiência técnica, eficiência alocativa

Abstract: This study measured levels of total factor productivity and technical, allocative and economic efficiency in agricultural crop production for Brazil in 2006. A nonparametric frontier model (DEA) under constant returns to scale was used. On average, the results suggest that the sector suffers from moderate technical inefficiency and from strong allocative inefficiency. If full technical efficiency were achieved, the crop production would increase by $5 \%$ over that obtained in 2006. It has been found overuse of the labor. São Paulo States agriculture was the only operating in full efficiency in 2006. The data indicated a catch up process in the productivity and efficiency indexes in 2006 compared to 1995.

Keywords: agricultural supply, total factor productivity, economic efficiency, technical efficiency, allocative efficiency

\footnotetext{
Recebido em: 6/9/11; Aceito em: 27/2/2012.

O autor agradece a colaboração de José G. Gasques nas fases iniciais da pesquisa, as sugestões de Steven M. Helfand e as críticas de Eliane G. Gomes e Geraldo S. Souza a uma versão anterior.

2 Doutor em Economia, Pesquisador do Instituto de Economia Agrícola (IEA). E-mail: jrvicente@iea.sp.gov.br.
} 


\section{Introdução}

Em 1995, a média nacional dos índices de eficiência revelou ineficiência técnica moderada na produção agrícola, setor de lavouras (VICENTE, 2004a). Os índices de eficiência econômica nas Unidades da Federação (UFs) estiveram condicionados pela eficiência alocativa, que apresentou, na quase totalidade dos casos, índices menores do que os de eficiência técnica. Somente o estado de São Paulo não apresentava algum grau de ineficiência econômica. Caso todas as UFs estivessem sobre a fronteira de eficiência técnica, a quantidade produzida em nível nacional poderia ter superado em mais de $30 \%$ a efetivamente obtida.

Entre 1970 e 1995, o estado de São Paulo deteve os mais elevados índices de produtividade, aumentando a distância em relação aos seguidores mais diretos, e diferenças significativas de produtividade foram encontradas entre as regiões geográficas (VICENTE, 2006). De 1995 a 2006, a produtividade total de fatores (PTF) da agricultura paulista, setor de lavouras, cresceu de $26 \%$ a $31 \%$, de acordo com resultados de medidas baseadas, respectivamente, em índices de Malmquist e índices de Fisher (VICENTE, 2008).

Com a divulgação dos resultados do Censo Agropecuário de 2006, realizado pelo Instituto Brasileiro de Geografia e Estatística (IBGE), podese verificar se as tendências observadas nos indicadores de PTF no período de 1970 a 1995 e de eficiência econômica no ano de 1995 permanecem ou se foram alteradas. Com a evolução dos meios de comunicação desde meados da década de 1990 e a maior disponibilidade de informações técnico-científicas para todos os agentes relacionados às cadeias de produção agrícolas, inclusive pela internet, e também com a ampliação e consolidação crescentes da Embrapa, é provável que as diferenças de produtividade e de eficiência entre as UFs estejam diminuindo. 
Análises de eficiência complementam estudos sobre produtividade de fatores, fornecendo medidas de desempenho que permitem avaliar as unidades produtivas, possibilitam identificar determinantes de ineficiência e subsidiar atividades de extensão, pesquisa e assistência técnica. Medidas de eficiência econômica podem também esclarecer aspectos da modernização da agricultura e do crescimento da produtividade; por exemplo, se as UFs que apresentam os maiores índices de produtividade são também as mais eficientes e se existe produção eficiente em regiões de agricultura mais tradicional.

Para subsidiar respostas e essas questões, o objetivo do presente estudo é estimar a produtividade total de fatores e a eficiência econômica na produção agrícola brasileira, setor de lavouras, em nível de UF, utilizando dados do Censo Agropecuário de 2006. Adicionalmente, pretende-se identificar casos de subutilização e sobreutilização de fatores de produção.

\section{Metodologia}

Considere-se uma firma produzindo $y$ com o uso dos insumos $x_{1}$ e $x_{2}$ e assuma-se que a função de produção de fronteira seja $y=f\left(x_{1}, x_{2}\right)$. Sob retornos constantes à escala, pode-se escrever $l=f\left(x_{1}, x_{2}\right)$, ou seja, a fronteira tecnológica pode ser representada por uma isoquanta unitária. Como a isoquanta $U U^{\prime}$ (Figura 1) não é observável, tem que ser estimada a partir de uma amostra de pontos, inclusive os ineficientes, como o representado pelo ponto A.

Se o ponto A, que, por definição não pode estar abaixo da curva $U U$, representa a produção da quantidade $y^{o}$ com o uso de $x_{1}^{o}$ e $x_{2}^{o}$, então a razão $\mathrm{OB} / \mathrm{OA}$ - que é a divisão dos insumos necessários para produzir $y^{\circ}$ pela quantidade de insumos efetivamente utilizada para produzir essa mesma quantidade $y^{o}$ - mede a ineficiência técnica. A isoquanta $U U^{\prime}$ representa, portanto, a produção mais eficiente em relação ao uso de insumos, e os desvios em relação a ela foram considerados por Farrell (1957) associados à ineficiência técnica das firmas. 


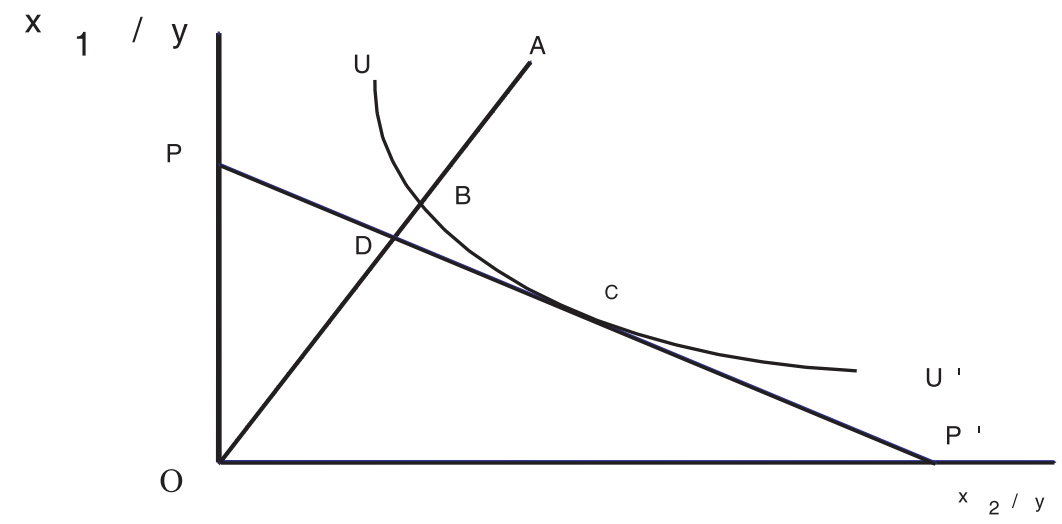

Figura 1 - Eficiências Técnica, Alocativa e Econômica.

Se a reta $P P^{\prime}$ representar os preços relativos dos insumos, a razão OD/ OB mede a ineficiência alocativa, com os custos no ponto $\mathrm{D}$ sendo os mesmos do ponto $\mathrm{C}$, alocativamente eficiente; já os custos no ponto $\mathrm{B}$, que é tecnicamente eficiente, são maiores do que os dos pontos $\mathrm{D}$ e $\mathrm{C}$, devido à ineficiência alocativa. Uma medida da eficiência total (ou eficiência econômica) é proporcionada pela relação OD/OA. Essa formulação é conhecida como modelo de eficiência-custo de FarrellDebreu (COOPER, SEIFORD e TONE, 2007).

\subsection{Análise Envoltória de Dados (DEA) e Nova Eficiência-Custo}

A DEA é uma alternativa de construção de fronteiras de melhor prática, sem necessidade de especificação da tecnologia de produção, proposta por Charnes, Cooper e Rhodes (1978). A eficiência de uma determinada "unidade tomadora de decisões (DMUs)" é medida em relação a todas as outras unidades com a restrição simples de que todas elas se encontram abaixo da fronteira eficiente ou, no máximo, sobre ela (SEIFORD e THRALL, 1990). 
A definição de DMU é genérica e flexível, e ao longo dos anos ocorreu uma grande variedade de aplicações de DEA na avaliação do desempenho dos diferentes tipos de entidades envolvidas em atividades, contextos e países diferentes. Estas aplicações avaliaram o desempenho de entidades como hospitais, setores governamentais, universidades, cidades, tribunais, empresas e outros, incluindo o desempenho dos países, regiões etc. Por exigir muito poucas hipóteses, a DEA também abriu possibilidades para o uso em casos resistentes a outras abordagens devido à natureza complexa e muitas vezes desconhecida das relações entre os múltiplos insumos e os múltiplos produtos envolvidos (COOPER, SEIFORD e ZHU, 2004).

Mais especificamente, trata-se de uma metodologia de programação linear que utiliza dados de quantidades de produto e de insumos na construção de uma superfície linear compreensível. A superfície de fronteira é obtida pela resolução de uma sequência de problemas de programação linear, um para cada observação (DMU) da amostra (RAO e COELLI, 1999). O grau de ineficiência de cada DMU é obtido pela distância de cada ponto até a fronteira. No presente estudo, foi utilizado um modelo DEA insumo-orientado, que define a fronteira procurando a máxima redução proporcional no uso dos insumos, mantendo constante o nível de produto de cada observação.

Utilizando o teorema da dualidade, o problema de programação matemática, considerando-se retornos constantes à escala, pode ser representado por (COELLI, 1996):

$$
\min _{\theta, \lambda} \theta
$$

sujeito às restrições:

$-\mathrm{y}_{\mathrm{i}}+\mathrm{Y} \lambda \geq 0$,

$\theta \mathrm{x}_{\mathrm{i}}-\mathrm{X} \lambda \geq 0$,

$\lambda \geq 0$, 
em que $\theta \leq 1$ é um escalar representativo do grau de eficiência técnica (ET) da $i$-ésima DMU e $\lambda$ é um vetor (Nx1) de constantes. Um valor de $\theta=1$ indica um ponto sobre a fronteira, ou seja, uma DMU eficiente de acordo com a definição de Farrell (1957). No caso de uma DMU eficiente, todos os valores de $\lambda$ serão iguais a zero; para as DMUs ineficientes, os valores de $\lambda$ são os pesos utilizados na combinação de DMUs eficientes que projetam cada DMU ineficiente sobre a fronteira. Esse problema de programação linear tem que ser resolvido $\mathrm{N}$ vezes, uma para cada observação da amostra.

Dispondo-se de informações sobre preços de insumos, podem-se mensurar eficiências técnica e alocativa pela resolução do seguinte modelo DEA de minimização de custo (COELLI, 1996):

$\min _{\lambda, x_{i}^{*}} \mathrm{~W}_{\mathrm{i}}{ }^{\prime} \mathrm{x}_{\mathrm{i}}^{*}$

sujeito às restrições:

$-\mathrm{y}_{\mathrm{i}}+\mathrm{Y} \lambda \geq 0$

$\mathrm{x}_{\mathrm{i}}^{*}-\mathrm{X} \lambda \geq 0$,

$\lambda \geq 0$,

em que $\mathrm{w}_{\mathrm{i}}$ é o vetor de preços de insumos para a i-ésima DMU e $\mathrm{x}_{\mathrm{i}}{ }^{*} \mathrm{e}$ o vetor de quantidades de insumos que minimizam os custos para a $i$ ésima DMU, dados os preços dos insumos $\left(\mathrm{w}_{\mathrm{i}}\right)$ e as quantidades de produto $\left(\mathrm{y}_{\mathrm{i}}\right)$.

A eficiência econômica (ou eficiência total, ou eficiência custo) da $i$ ésima DMU pode ser calculada por (COELLI, 1996):

$\mathrm{EE}_{\mathrm{i}}=\mathrm{w}_{\mathrm{i}}^{\prime} \mathrm{x}_{\mathrm{i}}^{*} / \mathrm{w}_{\mathrm{i}}^{\prime} \mathrm{x}_{\mathrm{i}}$ 
ou seja, pela razão entre o custo mínimo e o custo observado.

A eficiência alocativa (EA) é obtida residualmente:

$\mathrm{EA}_{\mathrm{i}}=\mathrm{EE}_{\mathrm{i}} / \mathrm{ET}_{\mathrm{i}}$

em que $\mathrm{ET}_{\mathrm{i}}$ é a eficiência técnica da i-ésima DMU.

Indicadores de subutilização e superutilização de insumos derivam diretamente dos cálculos de eficiência anteriores. A produção potencial (de fronteira), mantido o nível atual de uso de insumos em cada UF, pode ser estimada através de (RAMANATHAN, 2000):

$\mathrm{PP}_{\mathrm{i}}=\mathrm{PA}_{\mathrm{i}} / \mathrm{ET}_{\mathrm{i}}$

em que $\mathrm{PP}_{\mathrm{i}}$ é a produção potencial da $i$-ésima $\mathrm{DMU}$ e $\mathrm{PA}_{\mathrm{i}}$ é a produção atual.

Kaoru Tone mostrou, em 2002, que o modelo de eficiência-custo de FarellDebreu - nos casos em que os preços unitários dos insumos não sejam idênticos entre as DMUs - pode indicar eficiências custo e alocativas idênticas para duas DMUs quaisquer, apesar de uma delas apresentar custos maiores do que a outra, o que não é aceitável (COOPER, SEIFORD e TONE, 2007).

A solução proposta baseia-se em um novo conjunto de possibilidades de produção que considera explicitamente o custo unitário dos insumos e que possibilita medir uma nova eficiência técnica obtida como a solução ótima do seguinte problema de programação linear (COOPER, SEIFORD e TONE, 2007):

[NTech $] \bar{\theta}^{*}=\min _{\bar{\theta}, \lambda} \bar{\theta}$

sujeito às restrições: 


$$
\begin{aligned}
& \bar{\theta} \bar{x}_{0} \geq \bar{X} \lambda \\
& y_{0} \leq Y \lambda \\
& \lambda \geq 0
\end{aligned}
$$

A nova eficiência-custo (ou nova eficiência econômica) é definida por:

$$
\bar{\gamma}^{*}=\overline{e x}_{0}^{*} / \overline{e x}_{0}
$$

em que $e \in R^{m}$ é um vetor linha com todos os elementos iguais a $1 \mathrm{e} \bar{x}_{0}^{*}$ é a solução ótima para o problema de programação linear:

$$
[\mathrm{NCost}] \mathrm{e}_{0}^{*}=\min _{\bar{x}, \lambda} \mathrm{e} \overline{\mathrm{x}}
$$

sujeito às restrições:

$$
\begin{aligned}
& \bar{x} \geq \bar{X} \lambda \\
& y_{0} \leq Y \lambda \\
& \lambda \geq 0
\end{aligned}
$$

Essa nova medida elimina a ocorrência do fenômeno anteriormente descrito, conforme demonstrado em Cooper, Seiford e Tone (2007).

\subsection{Fontes de dados e variáveis utilizadas na estimação da fronteira}

Para representar a produção, tentou-se utilizar, inicialmente, as informações do Censo Agropecuário de 2006, disponíveis no site do IBGE (http://www.ibge.gov.br/home/ estatistica/economia/agropecuaria/ censoagro/brasil_2006/default.shtm). Entretanto, testes de depuração 
simples cruzando informações (cálculo de rendimentos de lavouras e de preços médios de produtos) revelaram inconsistências e aquela fonte foi abandonada, optando-se por empregar os resultados da Pesquisa Agrícola Municipal (PAM) para 2006, também disponíveis no site do IBGE (ftp:/ / f t p.ibge.gov.br/Produca o_Agricola/Produca Agricola_Municipal_\%5Banual\%5D/2006/). Em alguns poucos casos em que os dados da PAM apresentaram também inconsistências claras em preços ou rendimentos de lavouras, voltou-se ao Censo Agropecuário para tentar corrigi-las.

Os produtos de lavouras permanentes considerados foram: abacate, algodão arbóreo, banana, borracha, cacau, café, caqui, castanha de caju, chá-da-índia, coco-da-baía, dendê, erva-mate, figo, goiaba, guaraná, laranja, limão, maçã, mamão, manga, maracujá, marmelo, noz, palmito, pêra, pêssego, pimenta-do-reino, sisal ou agave, tangerina, tungue, urucum e uva. De lavouras temporárias, foram considerados os produtos: abacaxi, algodão herbáceo, alho, amendoim, arroz, aveia, batata-doce, batatainglesa, cana-de-açúcar, cebola, centeio, cevada, ervilha, fava, feijão, fumo, girassol, juta, linho, malva, mamona, mandioca, melancia, melão, milho, rami, soja, sorgo granífero, tomate, trigo e triticale.

A produção agrícola, em nível de UF, foi representada por um único índice de quantidade, calculado por meio de uma fórmula agregativa ponderada. Foi construído um índice de desempenho, tomando-se os totais do País (somas das quantidades das UFs = quantidades do Brasil e somas dos valores das UFs divididas pelas respectivas somas das quantidades = preços médios do Brasil) como base. Isso conduz ao "problema dos números-índice", ou da escolha de determinada fórmula mais adequada para representar o problema em questão. Tradicionalmente, a comparação entre as diversas fórmulas existentes era feita com base nos testes lógicos definidos por Fisher em 1922 (enfoque axiomático).

Desde a década de 1970, vem merecendo destaque o estudo das relações entre especificações funcionais admitidas pela análise econômica e fórmulas de números-índice, no chamado enfoque econômico. Diewert 
(1976) chamou de superlativa uma fórmula de números-índice exata (isto é, consistente) para uma forma funcional flexível e demonstrou que o índice translog de Törnqvist-Theil e o índice ideal de Fisher são superlativos. Como as medidas provenientes de fórmulas superlativas são próximas, a questão da escolha entre elas se torna menos relevante.

Devido às suas reconhecidas vantagens, optou-se por utilizar a fórmula ideal de Fisher, que pode ser representada formalmente, para cada UF, por:

$$
\mathrm{FQ}_{0,1}=\sqrt{\frac{\sum_{\mathrm{i}=1}^{\mathrm{n}} \mathrm{p}_{0}^{\mathrm{i}} \mathrm{q}_{1}^{\mathrm{i}}}{\sum_{\mathrm{i}=1}^{\mathrm{n}} \mathrm{p}_{0}^{\mathrm{i}} \mathrm{q}_{0}^{\mathrm{i}}} \cdot \frac{\sum_{\mathrm{i}=1}^{\mathrm{n}} \mathrm{p}_{1}^{\mathrm{i}} \mathrm{q}_{1}^{\mathrm{i}}}{\sum_{\mathrm{i}=1}^{\mathrm{n}} \mathrm{p}_{1}^{\mathrm{i}} \mathrm{q}_{0}^{\mathrm{i}}}}
$$

em que FQ identifica o índice Fisher de quantidade produzida na UF em questão, $p_{1}^{i}$ é o preço do $i$-ésimo produto nessa UF, $p_{0}^{i}$ o preço do $i$ ésimo produto em nível nacional, $q_{1}^{i}$ a quantidade do i-ésimo produto na UF em questão e $q_{0}^{i}$, a quantidade do $i$-ésimo produto em nível nacional. O primeiro quociente dentro da raiz é um índice Laspeyres, e o segundo quociente, um índice Paasche. Para representar índices de preço calculados pela fórmula ideal de Fisher, basta substituir $q$ por $p$, e viceversa, em (9).

A PTF, em nível de UF, foi obtida pelo quociente do índice de quantidade de produto pelo respectivo índice de uso de fatores de produção. Conforme Alves (2010), é uma medida fundamental porque empresta significado à parcela de crescimento da produção não explicada pelo aumento no uso dos insumos. 
Os fatores de produção considerados foram: terra (área colhida), trabalho (pessoal ocupado, soma de responsáveis e membros da família, empregados permanentes, empregados temporários, parceiros e outras condições), mecanização (máquinas e combustíveis), fertilizantes, defensivos e os investimentos em culturas perenes. A área colhida teve como fonte, basicamente, a PAM do IBGE, como descrito anteriormente. O pessoal ocupado foi obtido do Censo Agropecuário de 2006 e se refere aos grupos econômicos lavouras temporárias, lavouras permanentes e horticultura e floricultura. Valores de arrendamento e salários de empregados permanentes tiveram como fonte os bancos de dados da Função Getúlio Vargas (FGV) e do Instituto de Economia Agrícola (IEAAPTA). As quantidades e preços de defensivos utilizados (toneladas de princípio-ativo) foram provenientes de planilhas calculadas, em nível de UF, pelo Sindicato Nacional da Indústria de Produtos para Defesa Agrícola (SINDAG). A publicação da Associação Nacional para a Difusão de Adubos e Corretivos Agrícolas (ANDA) forneceu as quantidades de fertilizantes (toneladas de nutrientes) utilizadas nas UFs (ANUÁRIO, 2007); os preços foram calculados a partir das despesas com adubos e corretivos do solo declaradas no Censo Agropecuário de 2006, também referentes aos grupos econômicos lavouras temporárias, lavouras permanentes e horticultura e floricultura (preço $=$ despesa/quantidade).

Para o fator mecanização, partiu-se dos estoques de tratores e colhedoras existentes nos estabelecimentos rurais, referentes aos três grupos econômicos relacionados às lavouras. Os preços das máquinas foram obtidos pelos valores de veículos, tratores, máquinas e implementos declarados no Censo Agropecuário de 2006, divididos pelo número de tratores e colhedoras. Esses preços dos estoques foram transformados em fluxos de serviços utilizando a fórmula $R_{i}=r V_{i}^{T_{i}} / 1-e^{-r T_{i}}$, desenvolvida por Yotopoulos em 1967 (VICENTE, 2006), em que $R_{i}$ é o fluxo anual constante de serviços do i-ésimo ativo, $V_{i}^{T i}$ é seu preço, $T_{i}$ é sua expectativa de vida (assumida como igual a 20 anos) e $r$ é a taxa de desconto $(8,4 \%$, igual à da caderneta de poupança em 2006). Os combustíveis foram representados pelas despesas com combustíveis 
declaradas no Censo Agropecuário e pelos preços médios do óleo diesel (quantidade $=$ despesas/preço).

O fator investimentos em culturas perenes teve como base o valor das lavouras permanentes declarado no Censo Agropecuário de 2006. Os valores desses estoques foram transformados em fluxos de serviços também através da fórmula de Yotopoulos, assumindo-se uma vida útil de 10 anos, e a mesma taxa de desconto utilizada para a mecanização. As quantidades desse fator foram representadas pelas áreas ocupadas pelas culturas permanentes, e os preços foram obtidos dividindo-se os fluxos de valores totais pelas áreas.

Ressalte-se que os mesmos problemas encontrados nos preços calculados dos produtos ocorreram com os preços calculados para as máquinas e, em menor escala, para os de fertilizantes, para os de óleo diesel e para os de lavouras perenes. Todavia, não foi encontrada fonte alternativa para substituir essas informações.

O modelo DEA utilizado para o cálculo de eficiência foi especificado com um produto (índice de quantidade produzida, em cada UF, em relação ao total nacional) e seis insumos: terra (área colhida em cada UF), trabalho (número de pessoas empregadas em cada UF), mecanização (estoque de tratores e colhedoras em cada UF), fertilizantes (quantidade utilizada em cada UF), defensivos (quantidade utilizada em cada UF) e investimentos em culturas perenes (área com culturas permanentes em cada UF). Os preços considerados para esses insumos foram descritos anteriormente. No caso da mecanização, foram somados os valores das quantidades médias de combustíveis utilizados, por máquina, aos preços dos fluxos de serviços, em cada UF.

Face às características dos fatores de produção descritos - em sua quase totalidade divisíveis e neutros à escala, especialmente ao nível de UF - não foi imposta a restrição de retornos variáveis à escala aos modelos DEA, procedimento que tenderia à indicação de mais DMUs como tecnicamente eficientes. A imposição injustificada de retornos variáveis à escala em modelos DEA é um erro de especificação que deve ser evitado (SMITH, 1997). 
A opção por um modelo de retornos constantes à escala levou ao emprego da fórmula de Laspeyres no cálculo do índice de desempenho utilizado para representar o produto ${ }^{3}$. Esse índice indica o percentual de quantidade produzida, em cada uma das UFs, em relação ao total nacional, considerando-se para todas as observações os preços médios em nível nacional. Equivale exatamente a transformar os dados de output das DMUs em proporções ou percentuais do total de todas as DMUs da amostra para essa variável. Os modelos DEA do tipo CCR, desenvolvido por Charnes, Cooper e Rhodes (1978) - com retornos constantes à escala - são invariáveis a mudanças nas unidades de medida adotadas, ou seja, os resultados não se alteram caso uma ou mais variáveis sejam divididas ou multiplicadas por uma ou mais constantes (COELLI, 1996; COOPER, SEIFORD e TONE, 2007).

Portanto, o índice de desempenho aqui empregado não se enquadra no caso levantado por Hollingsworth e Smith (2003), em que o uso de quocientes em modelos DEA levaria a incorreções no modelo de retornos constantes, sendo mais adequado o de retornos variáveis.

\section{Resultados e Discussão}

No ano de 2006 , sete UFs concentraram cerca de $80 \%$ da quantidade produzida ${ }^{4}$ e responderam individualmente por percentuais superiores a $5 \%$ da produção total brasileira de lavouras: São Paulo (22,47\%), Paraná (12,87\%), Minas Gerais (11,59\%), Rio Grande do Sul (10,75\%), Mato Grosso $(8,59 \%)$, Bahia $(7,91 \%)$ e Goiás $(5,76 \%)$ (Tabela 1). A rigor, como o índice ideal de Fisher não é transitivo, não se podem comparar UFs com o procedimento adotado, apenas cada uma das UFs com o total nacional, pois comparações entre UFs exigiriam o uso de um método multilateral como o EKS utilizado, por exemplo, por Vicente (2004b).

\footnotetext{
O índice de Laspeyres é conceitualmente inferior ao índice ideal de Fisher. Não obstante, no caso em análise, as duas medidas de produção agrícola estiveram bem próximas, com coeficiente de correlação igual a 0,9977.

4 Os dados referentes à produção (quantidade produzida) discutidos resultam do índice ideal de Fisher. Como citado anteriormente, os resultados do índice Laspeyres foram utilizados apenas para obter as medidas de eficiência.
} 
Tabela 1 - Índices de Desempenho e de Eficiência (Técnica, Alocativa e Econômica), Unidades da Federação, Brasil, 2006.

\begin{tabular}{|c|c|c|c|c|c|c|c|}
\hline \multirow[b]{2}{*}{ Unidade da Federação } & \multicolumn{3}{|c|}{ Índice de Desempenho $\left({ }^{\mathrm{l}}\right)$} & \multicolumn{4}{|c|}{ Índice de Eficiência $\left({ }^{2}\right)$} \\
\hline & produção & fatores & PTF & produção( $\left.{ }^{3}\right)$ & técnica & alocativa & econômica \\
\hline Alagoas & 1,21 & 1,41 & 86,17 & 1,16 & 1,00 & 0,51 & 0,51 \\
\hline Bahia & 7,91 & 8,39 & 94,31 & 8,00 & 1,00 & 0,53 & 0,53 \\
\hline Ceará & 1,45 & 2,67 & 54,39 & 1,52 & 0,85 & 0,56 & 0,47 \\
\hline Paraíba & 0,88 & 1,01 & 87,26 & 0,89 & 1,00 & 0,65 & 0,65 \\
\hline Pernambuco & 1,95 & 2,33 & 83,74 & 1,83 & 1,00 & 0,49 & 0,49 \\
\hline Piauí & 0,63 & 1,91 & 33,23 & 0,63 & 0,43 & 0,58 & 0,25 \\
\hline Rio Grande do Norte & 0,40 & 0,65 & 61,74 & 0,41 & 0,81 & 0,56 & 0,45 \\
\hline Sergipe & 0,48 & 0,67 & 71,27 & 0,48 & 0,87 & 0,52 & 0,45 \\
\hline Maranhão & 1,27 & 2,90 & 43,71 & 1,29 & 0,69 & 0,52 & 0,36 \\
\hline Acre & 0,15 & 0,21 & 69,97 & 0,17 & 1,00 & 0,54 & 0,54 \\
\hline Amapá & 0,03 & 0,05 & 53,40 & 0,02 & 0,92 & 0,49 & 0,45 \\
\hline Amazonas & 0,32 & 0,80 & 40,32 & 0,37 & 1,00 & 0,35 & 0,35 \\
\hline Pará & 0,94 & 2,38 & 39,37 & 1,00 & 0,63 & 0,54 & 0,33 \\
\hline Roraima & 0,09 & 0,11 & 80,08 & 0,10 & 1,00 & 0,90 & 0,90 \\
\hline Rondônia & 0,71 & 0,80 & 88,15 & 0,74 & 1,00 & 0,70 & 0,70 \\
\hline Tocantins & 0,56 & 0,73 & 75,91 & 0,59 & 1,00 & 0,51 & 0,51 \\
\hline Distrito Federal & 0,16 & 0,17 & 97,98 & 0,16 & 0,98 & 0,63 & 0,62 \\
\hline Goiás & 5,76 & 5,34 & 108,03 & 5,54 & 1,00 & 0,88 & 0,88 \\
\hline Mato Grosso & 8,59 & 9,76 & 88,02 & 9,42 & 1,00 & 0,62 & 0,62 \\
\hline Mato Grosso do Sul & 2,97 & 3,06 & 97,32 & 2,97 & 1,00 & 0,62 & 0,62 \\
\hline Minas Gerais & 11,59 & 10,33 & 112,28 & 11,20 & 1,00 & 0,76 & 0,76 \\
\hline Espírito Santo & 1,46 & 1,69 & 86,18 & 1,60 & 1,00 & 0,49 & 0,49 \\
\hline Rio de Janeiro & 0,44 & 0,45 & 97,77 & 0,44 & 0,97 & 0,55 & 0,53 \\
\hline São Paulo & 22,47 & 13,81 & 162,65 & 21,46 & 1,00 & 1,00 & 1,00 \\
\hline Paraná & 12,87 & 13,17 & 97,73 & 12,25 & 0,84 & 0,78 & 0,65 \\
\hline Santa Catarina & 3,93 & 3,53 & 111,43 & 4,14 & 1,00 & 0,78 & 0,78 \\
\hline Rio Grande do Sul & 10,75 & 11,65 & 92,26 & 11,62 & 1,00 & 0,75 & 0,75 \\
\hline Brasil $\left({ }^{4}\right)$ & 100,00 & 100,00 & 100,00 & 100,00 & 0,91 & 0,61 & 0,55 \\
\hline
\end{tabular}

(1) Índices de desempenho calculados pela fórmula ideal de Fisher (base: Brasil=100).

${ }^{2}$ ) Índices de nova-eficiência de Tone (COOPER, SEIFORD e TONE, 2007).

${ }^{(3)}$ Índice Laspeyres de quantidade produzida, utilizado no cálculo dos índices de eficiência.

$\left({ }^{4}\right)$ Para o Brasil, os índices de eficiência são as médias geométricas, e os de quantidade produzida e de uso de fatores são as somas das Unidades da Federação.

Fonte: Dados da pesquisa. 
A produtividade total de fatores (PTF) no setor de lavouras variou de 33,23 (Piauí) a 162,65 (São Paulo), tomando-se o Brasil como base $(=100)^{5}$. Embora esses valores aparentem grande dispersão, deve-se salientar que esses resultados indicam um processo de convergência entre os índices de PTF. Em 1995, de acordo com os cálculos de Vicente, Anefalos e Caser (2001) - também utilizando índices de Fisher - a amplitude de variação era ainda maior: o índice do Piauí foi igual a 18,29 e o de São Paulo igual a 227,07 (Brasil=100).

O estado da Bahia liderou a PTF na região Nordeste em 2006, com índice $(94,31)$ próximo da média nacional (100). Em seguida, com índices superiores a 80, apareceram os estados da Paraíba, Alagoas e Pernambuco, e índices inferiores a 50 foram estimados para os estados do Piauí e do Maranhão (Tabela 1).

Na região Norte, Rondônia e Roraima apresentaram os maiores níveis de PTF, 88,15 e 80,08, respectivamente, enquanto os índices do Pará e do Amazonas foram menores do que 50 (Tabela 1).

Nas regiões Centro-Oeste, Sudeste e Sul, os índices estimados para todas as UFs foram superiores a 80, destacando-se os estados de Goiás $(108,03)$, Minas Gerais $(112,28)$ e Santa Catarina $(111,43)$ que, assim como São Paulo $(162,65)$, exibiram níveis de produtividade (PTF) superiores à média nacional (Tabela 1).

O valor da média geométrica dos índices de eficiência econômica calculados para as UFs - média nacional - resultou em 0,55. As médias das regiões Sul $(0,73)$, Centro-Oeste $(0,68)$ e Sudeste $(0,66)$ foram superiores à média nacional; as UFs das regiões Norte e Nordeste apresentaram as menores médias, respectivamente, 0,51 e 0,45. Esses resultados estiveram associados aos níveis do índice de eficiência alocativa, e apenas o estado do Piauí teve índice de eficiência alocativa superior ao de eficiência técnica (Tabela 1).

A evolução da PTF em nível de UF, no período 1970 a 2006 pode ser vista em Gasques et al. (2010). 
Apesar de o estado de São Paulo continuar sendo o único com índice de eficiência econômica igual a 1, os resultados desses índices, assim como os de PTF, apontam para um movimento de convergência: em 1995, o Piauí teve índice igual a 0,07 e a média nacional foi 0,34 (VICENTE, 2004a), enquanto em 2006 o índice do Piauí passou para 0,25 e a média nacional para 0,55 .

O coeficiente de correlação entre os índices de PTF e de eficiência econômica foi igual a 0,848 ; dessa forma, um modelo de regressão simples relacionando essas duas variáveis teria coeficiente de determinação igual a $71,9 \%$. Portanto, as variações de um desses índices "explicariam" mais de $70 \%$ das variações do outro índice.

Ray e Bhadra (1993) empregaram um esquema para categorizar violações, em nível de firma, do axioma fraco de minimização de custos. Com base nesse esquema, Pereira $\mathrm{F}^{\mathrm{o}}$ e Ferreira $\mathrm{F}^{\mathrm{o}}(2003)$ classificaram índices de eficiência em ineficiência fraca $(0,9<\mathrm{EE}<1,0)$, ineficiência moderada $(0,7<\mathrm{EE}<0,9)$ e ineficiência forte $(\mathrm{EE}<0,7)$, podendo-se adicionar a classe sem ineficiência $(E E=1)$. Por essa classificação, os estados de Roraima, Rondônia, Goiás, Santa Catarina, Minas Gerais e Rio Grande do Sul mostravam ineficiência econômica moderada; as demais UFs, ineficiência econômica forte (Tabela 1).

Em nível nacional, a média do índice de eficiência técnica foi igual a 0,91, quase igual à da região Norte $(0,92)$, superada pelas regiões Centro Oeste $(1,00)$, Sudeste $(0,99)$ e Sul $(0,94)$. Já a média nacional do índice de eficiência alocativa $(0,61)$ foi superada pelas médias das regiões $\operatorname{Sul}(0,77)$, Centro-Oeste $(0,68)$ e Sudeste $(0,66)$.

As unidades da Federação com índices de eficiência técnica em patamar superior a $0,90 \mathrm{em}$ todas as regiões geográficas mostram a existência simultânea de produção tecnicamente eficiente em UFs caracterizadas por agricultura bem estabelecida e modernizada, em UFs em rápido processo de modernização e também em UFs com explorações comparativamente mais tradicionais. 
Os índices de eficiência alocativa, por outro lado, mostram ineficiência forte em UFs de quase todas as regiões do País. Apenas São Paulo (sem ineficiência), Roraima, Goiás, Santa Catarina, Paraná, Minas Gerais, Rio Grande do Sul e Rondônia (com ineficiência moderada) aparecem com padrões superiores.

Tomando-se como base os índices de eficiência técnica estimados e projetando-se as produções para a fronteira de melhor prática com o mesmo nível de uso de fatores, foi possível concluir que a quantidade produzida em 2006, em nível nacional, poderia ter sido 4,9\% maior, considerando-se o índice de Laspeyres, e 5,0\% maior, tomando-se o índice ideal de Fisher. Nas regiões geográficas, somente as regiões Sudeste e Centro-Oeste manteriam os mesmos níveis de quantidades produzidas observados, com as demais podendo apresentar índices superiores: 20,5\% na Norte; $11,4 \%$ na Nordeste; e $9,2 \%$ na Sul.

Do índice de eficiência alocativa, pode ser derivado outro indicador relacionado à subutilização ou sobreutilização de fatores, mantidos os mesmos níveis de produção. Foi construída uma escala do tipo Likert, que pode ser definida como uma escala com medidas categóricas baseadas em juízos de intensidade, ordenada e em oposição semântica em torno de um ponto médio. Foram atribuídos valor zero às recomendações de alteração de uso de fatores entre $\pm 30 \%$; valor -1 às recomendações de diminuição de uso maiores do que 30\% (sobreutilização); e valor +1 às recomendações de elevação de uso superiores a 30\% (subutilização).

A partir dos valores das UFs, foram calculadas médias regionais e nacionais, por sua vez interpretadas de acordo a escala proposta por Vicente (2004a): I<-0,75 sobreutilização forte; $-0,75 \leq \mathrm{I}<-0,50$ sobreutilização moderada; $-0,50 \leq \mathrm{I}<-0,25$ sobreutilização fraca; $0,25 \leq \mathrm{I} \leq 0,25$ utilização correta; $0,25<\mathrm{I} \leq 0,50$ subutilização fraca; $0,50<$ I $\leq 0,75$ subutilização moderada; e $0,75<$ I subutilização forte.

Em nível nacional, os resultados indicaram sobreutilização forte de trabalho, sendo que terra, mecanização e fertilizantes permaneceram na 
faixa de utilização correta, e os defensivos, na faixa de subutilização fraca (Tabela 2).

Diversos resultados regionais diferiram das médias nacionais: no Nordeste, sobreutilização fraca de terra, sobreutilização forte de trabalho, subutilização fraca de mecanização e subutilização moderada de defensivos; no Norte, sobreutilização forte de trabalho, subutilização moderada de fertilizantes e subutilização forte de defensivos.

Tabela 2 - Indicadores de Subutilização/Sobreutilização de Fatores nas Unidades da Federação, Brasil, 2006 ( $\left.^{1}\right)$

\begin{tabular}{|c|c|c|c|c|c|c|}
\hline \multirow[b]{2}{*}{ Unidade da Federação / Região } & \multicolumn{6}{|c|}{ Fator de Produção } \\
\hline & terra & trabalho & mecanização & fertilizantes & defensivos & $\begin{array}{l}\text { lavouras } \\
\text { perenes }\end{array}$ \\
\hline Alagoas & 0 & -1 & 0 & (1) & 1 & 1 \\
\hline Bahia & -1 & -1 & 1 & 0 & 0 & -1 \\
\hline Ceará & 0 & -1 & 1 & 1 & 1 & -1 \\
\hline Paraíba & 0 & -1 & 1 & 1 & 1 & 1 \\
\hline Pernambuco & 0 & -1 & 1 & 0 & 1 & -1 \\
\hline Piauí & -1 & -1 & -1 & 0 & 0 & 0 \\
\hline Rio Grande do Norte & 0 & -1 & 0 & 0 & 1 & -1 \\
\hline Sergipe & -1 & -1 & 1 & 1 & 1 & -1 \\
\hline Maranhão & -1 & -1 & 0 & 0 & 0 & 1 \\
\hline Nordeste (média) & $-0,44$ & $-1,00$ & 0,44 & 0,22 & 0,67 & $-0,22$ \\
\hline Acre & 0 & -1 & 0 & 1 & 1 & 1 \\
\hline Amapá & 0 & -1 & 1 & 1 & 1 & 0 \\
\hline Amazonas & 1 & -1 & 1 & 1 & 1 & 0 \\
\hline Pará & -1 & -1 & 0 & 1 & 1 & -1 \\
\hline Roraima & 1 & -1 & 1 & 1 & 1 & 1 \\
\hline Rondônia & 0 & -1 & -1 & 1 & 1 & -1 \\
\hline Tocantins & -1 & -1 & -1 & -1 & 0 & -1 \\
\hline Norte (média) & 0,14 & $-1,00$ & 0,14 & 0,71 & 0,86 & $-0,14$ \\
\hline Distrito Federal & 0 & -1 & -1 & -1 & -1 & 0 \\
\hline Goiás & 0 & 0 & 0 & 0 & -1 & 1 \\
\hline Mato Grosso & 0 & 1 & -1 & -1 & -1 & 1 \\
\hline Mato Grosso Sul & -1 & 0 & -1 & -1 & -1 & 0 \\
\hline Centro-Oeste (média) & $-0,25$ & 0,00 & $-0,75$ & $-0,75$ & $-1,00$ & 0,50 \\
\hline Minas Gerais & 1 & -1 & 0 & 0 & 0 & -1 \\
\hline Espírito Santo & -1 & -1 & 0 & 0 & 1 & -1 \\
\hline Rio de Janeiro & -1 & -1 & -1 & 1 & 1 & -1 \\
\hline São Paulo & 0 & 0 & 0 & 0 & 0 & 0 \\
\hline Sudeste (média) & $-0,25$ & $-0,75$ & $-0,25$ & 0,25 & 0,50 & $-0,75$ \\
\hline Paraná & -1 & -1 & -1 & 0 & 0 & 0 \\
\hline Santa Catarina & 0 & -1 & -1 & 0 & 1 & 1 \\
\hline Rio Grande do Sul & 0 & -1 & -1 & 0 & 0 & 1 \\
\hline Sul (média) & $-0,67$ & $-1,00$ & $-1,00$ & 0,00 & 0,33 & 0,67 \\
\hline Brasil (média) & $-0,22$ & $-0,81$ & $-0,07$ & 0,19 & 0,41 & $-0,07$ \\
\hline
\end{tabular}

(') Nas Unidades da Federação: -1 = sobreutilização; 0 = nível adequado; +1 = subutilização. As médias regionais e nacionais foram interpretadas de acordo com a escala explicitada no texto.

Fonte: Dados da pesquisa. 
No Centro-Oeste, sobreutilização moderada de mecanização e de fertilizantes, sobreutilização forte de defensivos e subutilização fraca de investimentos em culturas perenes; no Sudeste, sobreutilização moderada de trabalho, subutilização fraca de defensivos e sobreutilização moderada de investimentos em culturas perenes; e no Sul, sobreutilização fraca de terra, sobreutilização forte de trabalho e de mecanização e subutilização fraca de defensivos e subutilização moderada de investimentos em culturas perenes.

Essa aparente contradição entre sobreutilização forte de trabalho e de mecanização na região Sul pode estar associada aos problemas citados nos dados do Censo Agropecuário, ou ao método de transformação do estoque de máquinas em fluxo ou ainda ser um indicador de estoque excessivo de tratores e colhedeiras em relação ao uso efetivo.

\section{Conclusões e Considerações Finais}

A média nacional dos índices de eficiência revelou ineficiência técnica fraca na produção agrícola, setor de lavouras, em 2006. Eficiência técnica plena foi constatada, tanto em Unidades da Federação com agricultura moderna, quanto em outras UFs em processo de modernização ou com explorações mais tradicionais. Caso todas as UFs estivessem sobre a fronteira de eficiência técnica, a quantidade produzida, em nível nacional, poderia ter sido mais de $5 \%$ superior à efetivamente obtida.

Quanto à eficiência alocativa, a média nacional mostrou grau de ineficiência forte, resultado também verificado nas regiões, exceto no Sul, que apresentou ineficiência alocativa moderada. Simulações de minimização de custos indicaram, via de regra, sobreutilização de trabalho.

Os índices de eficiência econômica nas UFs estiveram geralmente condicionados pela eficiência alocativa, que apresentou índices menores do que os de eficiência técnica. Somente São Paulo não apresentou algum grau de ineficiência econômica. 
Tanto os índices de produtividade total de fatores quanto os de eficiência econômica tiveram amplitudes de variação menores dos que as ocorridas em 1995. As UFs com os menores índices de produtividade e eficiência estiveram mais próximas dos maiores níveis do que em 1995, o que pode ser indicador de um desejável processo de convergência.

\section{Referências}

ALVES, E. O que significam as medidas de produtividade da agricultura? Revista de Economia e Agronegócio, v. 8, n. 3, p. 349-370, set./dez. 2010.

CHARNES, A.; COOPER, W.; RHODES, E. Measuring the efficiency of decision making units. European Journal of Operational Research, v. 2, n. 6, p. 429-444, 1978.

COELLI, T. A Guide to DEAP version 2.1: a data envelopment analysis (computer) program. Armidale: Centre for Efficiency and Productivity Analysis, 1996.

COOPER, W. W.; SEIFORD, L. M.; TONE, K. Data envelopment analysis: a comprehensive text with models, applications, references and DEA-Solver software. New York: Springer, 2007

COOPER, W. W.; SEIFORD, L. M.; ZHU, J. Data envelopment analysis: models and interpretations, em Cooper, W. W.; Seiford, L. M.; Zhu, J. (organizadores) Handbook on data envelopment analysis. Boston: Kluwer Academic Publisher, 1-39, 2004.

DIEWERT, -W. E.--- Exact and superlative index numbers.- Journal of Econometrics, v. 4, n. 2, p. 115-145, may 1976.

FARRELL, M. J. The measurement of productive efficiency. Journal of Royal Statistical Society, A, n. 120 (Part 3), p. 253-281, 1957. 
GASQUES, J. G.; BASTOS, E. T.; BACHI, M. P. R.; VALDES, C. Produtividade total dos fatores e transformação da agricultura brasileira: análise de dados dos censos agropecuários. In: XLVIII CONGRESSO BRASILEIRO DE ECONOMIA, ADMINISTRAÇÃO E SOCIOLOGIA RURAL. Campo Grande: SOBER, 2010.

HOLLINGSWORTH, B.; SMITH, P. The use of ratios in data envelopment analysis. Applied Economics Letters, v.10, n.11, p. 733$735,2003$.

PEREIRA F $F^{\circ}$, C. A. E FERREIRA F ${ }^{\circ}$, J. B. S. Fontes de ineficiência da pequena produção familiar agrícola na região do Recôncavo do estado da Bahia. Revista de Economia e Sociologia Rural, v. 41 n. 1, p. $63-$ 78, jan./mar. 2003.

RAMANATHAN, R. Data envelopment analysis (DEA). Helsinki: Helsinki University of Technology, sep./dec. 2000. (Class Notes)

RAO, D. S. P.; COELLI, T. J. Economic growth, productivity change and inequality: methodology for the assessment of economic performance of nations. Armidale: Centre for Efficiency and Productivity Analysis, aug. 1999.

RAY, S. C.; BHADRA, D. Nonparametric tests of cost minimizing behavior: a study of Indian farms. American Journal of Agricultural Economics, v.75 n.4, p. 990-999, nov. 1993.

SEIFORD, L. M.; THRALL, R. M. Recent developments in DEA: the mathematical programming approach to frontier analysis. Journal of Econometrics, v. 46, n. 1/2, p. 7-38, oct./nov. 1990.

SMITH, P. Model misspecification in data envelopment analysis. Annals of Operations Research, v. 73, p. 233-252, oct. 1997.

VICENTE, J. R. Economic efficiency of agricultural production in Brazil. Revista de Economia e Sociologia Rural, v.42, n. 2, p. 201-232, abr./ jun. 2004a. 
VICENTE, J. R. Mudança tecnológica, eficiência e produtividade total de fatores na agricultura brasileira, 1970-95. Economia Aplicada, v. 8, n. 4, p. 729-760, out./dez. 2004b.

VICENTE, J. R. Comparação de produtividade agrícola entre Unidades da Federação, 1970-1995. Agricultura em São Paulo, v. 53 , n. 2, p. 69-83, 2006.

VICENTE, J. R. Produtividade total de fatores e eficiência econômica na agricultura paulista, 1995 a 2006. In: XL SIMPÓSIO BRASILEIRO DE PESQUISA OPERACIONAL João Pessoa: SOBRAPO, 2008.

VICENTE, J. R.; ANEFALOS, L. C.; CASER, D. V. Produtividade agrícola no Brasil, 1970-95. Agricultura em São Paulo, v. 48 , n. 2, p. 33-55, 2001. 